\title{
ON THE ASYMPTOTIC BEHAVIOR OF SOLUTIONS OF A NONLINEAR EQUATION
}

\author{
PAUL WALTMAN ${ }^{1}$
}

In [1] Moore and Nehari established the following: If $a(t)$ is positive and continuous, the equation

$$
x^{\prime \prime}+a(t) x^{2 n+1}=0, \quad n \text { a non-negative integer, }
$$

has solutions for which

$$
\lim _{t \rightarrow \infty} \frac{x(t)}{t}=\alpha>0
$$

if and only if

$$
\int^{\infty} t^{2 n+1} a(t) d t<\infty .
$$

This theorem is an extension of a theorem of Atkinson [2]. Theorem 1 of this paper establishes the sufficiency of the above theorem without an assumption as to the sign of $a(t)$. A theorem somewhat like the theorem of Trench [3] is given in Theorem 2 but for a nonlinear equation. As an application of this theorem, a stronger asymptotic result (under a stronger assumption) for equation (1) is given in Theorem 3. Other theorems on the asymptotic behavior of (1) when $a(t)>0$ are contained in [4].

Theorem 1. If $a(t)$ is continuous and

$$
\int^{\infty} t^{2 n+1}|a(t)| d t<\infty
$$

then equation (1) has a solution $x(t)$ with the property that

$$
\lim _{t \rightarrow \infty} \frac{x(t)}{t}=a \neq 0 .
$$

Proof. Let $A(t)=x^{\prime}(t), B(t)=x(t)-t x^{\prime}(t)$ so that

$$
x(t)=A(t) t+B(t)
$$

and

Received by the editors June 24, 1963.

1 This work was supported by the United States Atomic Energy Commission. Reproduction in whole or in part is permitted for any purpose of the U.S. Government. 


$$
t A^{\prime}(t)+B^{\prime}(t)=0 .
$$

$A(t)$ and $B(t)$ then are solutions of the system

$$
\begin{aligned}
-A^{\prime}(t) & =a(t)[A(t) t+B(t)]^{2 n+1}, \\
B^{\prime}(t) & =t a(t)[A(t) t+B(t)]^{2 n+1}
\end{aligned}
$$

or in integral form

$$
\begin{aligned}
& A(t)=A\left(t_{0}\right)-\int_{t_{0}}^{t} a(s) s^{2 n+1}\left[A(s)+\frac{B(s)}{s}\right]^{2 n+1} d s, \\
& B(t)=B\left(t_{0}\right)+\int_{t_{0}}^{t} a(s) s^{2 n+2}\left[A(s)+\frac{B(s)}{s}\right]^{2 n+1} d s .
\end{aligned}
$$

Note that the initial conditions on $A(t)$ and $B(t)$ are determined by the initial conditions on $x(t)$ and $x^{\prime}(t)$. Let $w(t)=A(t)+B(t) / t$ and denote $|A(t)|+|B(t) / t|$ by $\|w(t)\|$. Then from (3) it follows that $\left(t_{0}>0\right)$

$$
\|w(t)\| \leqq\left\|w\left(t_{0}\right)\right\|+2 \int_{t_{0}}^{t}|a(s)|\|w(s)\|^{2 n+1} s^{2 n+1} d s .
$$

In order to establish the boundedness of $\|w(t)\|$, the following theorem of Viswanatham [5] will be applied.

THEOREM 2. If $y(t) \leqq \eta+\int_{t}^{t} f(s, y(s)) d s$, where $f(t, y)$ is continuous and monotonic increasing in $y$ in the region $R$ defined by $\left|t-t_{0}\right| \leqq a$, $\left|y-y_{0}\right| \leqq b$, and $y(t)$ is continuous in the interval $\left|t-t_{0}\right| \leqq a$, then $y(t) \leqq \phi(t), t_{0} \leqq t \leqq t_{0}+\alpha$, where $\phi(t)$ is the maximal solution of the differential equation $z^{\prime}=f(x, z)$ through $\left(t_{0}, \eta\right)$ and $\alpha=\min [a, b / M]$, where $M$ is the bound on $|f(t, y)|$ in the given rectangle.

The differential equation associated with (4) is

$$
\begin{aligned}
\phi^{\prime} & =2|a(t)| t^{2 n+1} \phi^{2 n+1}(t), \\
\phi\left(t_{0}\right) & =\left\|w\left(t_{0}\right)\right\|
\end{aligned}
$$

which has solution $(n \neq 0)$

$$
\phi(t)=\left\{\left\|w\left(t_{0}\right)\right\|^{-2 n}-4 n \int_{t_{0}}^{t} s^{2 n+1}|a(s)| d s\right\}^{-1 / 2 n} .
$$

If

$$
k\left(t_{0}\right)=\int_{t_{0}}^{\infty}|a(t)| t^{2 n+1} d t
$$


then $\phi(t)$ will be bounded if the initial condition is chosen to satisfy

$$
\left\|w\left(t_{0}\right)\right\|<\frac{1}{\left[4 n k\left(t_{0}\right)\right]^{1 / 2 n}} .
$$

Although Theorem 2 is valid only locally, it can be extended in this case to arbitrarily large $t$ since $\phi(t)$ is known to exist for all $t$.

Suppose for $t_{1}>t_{0}$, it were the case that $\left\|w\left(t_{1}\right)\right\|>\phi\left(t_{1}\right)$. Then there is a last point $t_{2}<t_{1}$ where $\left\|w\left(t_{2}\right)\right\|=\phi\left(t_{2}\right)$ and Theorem 2 may be applied in a neighborhood of $t_{2}$.

From the form of $\phi(t)$, it can be seen that if a bound for $\phi(t)$ with $\phi\left(t_{0}\right)=\eta$ is found, this bound is more than sufficient for a solution with $\phi\left(t_{1}\right)=\eta, t_{1}>t_{0}$.

Given the boundedness of $\|w(t)\|$ it follows from equation (3) that $\lim _{t \rightarrow \infty} A(t)$ and $\lim _{t \rightarrow \infty} B(t) / t$, and thus $\lim _{t \rightarrow \infty} w(t)$, exist. However, $w(t)=x(t) / t$ and to prove the theorem there remains only to show that for some $x(t)$ this limit is not zero. This solution will be chosen by selecting appropriate initial conditions on $w(t)$. Let $\eta$ be a real number

$$
0<\eta<\left(\frac{1}{4 n k(0)}\right)^{1 / 2 n}
$$

and let $t_{0}$ be a number $>1$ such that

$$
k\left(t_{0}\right)<\frac{\eta}{\bar{w}^{2 n+1}},
$$

where $\bar{w}$ is the bound on $\|w(t)\|$ for $\|w(1)\|=\eta$, guaranteed by the above argument. Then the initial condition $A\left(t_{0}\right)=\eta, B\left(t_{0}\right)=0$ satisfies (4) and

$$
w(t)=\eta+\int_{t_{0}}^{t} a(s) s^{2 n+1}\left(\frac{s}{t}-1\right) w^{2 n+1} d s
$$

or

$$
w(\infty)>\eta-\bar{w}^{2 n+1} k\left(t_{0}\right)>0 .
$$

Thus there is a solution such that

$$
\lim _{t \rightarrow \infty} \frac{x(t)}{t}=\lim _{t \rightarrow \infty} w(t)=\alpha>0
$$

TheOREM 3. Let $z_{1}(t), z_{2}(t)$ be independent solutions of 


$$
z^{\prime \prime}=f(t) z
$$

and suppose $g(t)$ is continuous and that

$$
\int^{\infty}|g(t)| y(t) d t<\infty
$$

where

$$
y(t)=\max \left[z_{1}^{2 n+2}(t), z_{2}^{2 n+2}(t)\right] .
$$

Then there are solutions of

$$
u^{\prime \prime}=f(t) u+g(t) u^{2 n+1}
$$

which can be written in the form

$$
u(t)=\alpha(t) z_{1}(t)+\beta(t) z_{2}(t)
$$

where $\lim _{t \rightarrow \infty} \alpha(t)$ and $\lim _{t \rightarrow \infty} \beta(t)$ exist.

Proof. For convenience we shall suppose that the linearly independent solutions $z_{1}(t), z_{2}(t)$ have been chosen so that the Wronskian $z_{1}(t) z_{2}^{\prime}(t)-z_{2}(t) z_{1}^{\prime}(t)$ is equal to -1 . Let $A(t)=u^{\prime}(t) z_{2}(t)-u(t) z_{2}^{\prime}(t)$ and $B(t)=u(t) z_{1}^{\prime}(t)-u^{\prime}(t) z_{1}(t)$. Then

$$
\begin{aligned}
& u(t)=A(t) z_{1}(t)+B(t) z_{2}(t) \\
& A^{\prime}(t) z_{1}(t)+B^{\prime}(t) z_{2}(t)=0
\end{aligned}
$$

This yields the differential equations

$$
\begin{aligned}
& A^{\prime}(t)=g(t) z_{2}(t)\left[A(t) z_{1}(t)+B(t) z_{2}(t)\right]^{2 n+1}, \\
& B^{\prime}(t)=g(t) z_{1}(t)\left[A(t) z_{1}(t)+B(t) z_{2}(t)\right]^{2 n+1} .
\end{aligned}
$$

It is convenient to consider the integral form

$$
\begin{aligned}
& A(t)=A(0)+\int_{0}^{t} g(s) z_{2}(s)\left(A(s) z_{1}(s)+B(s) z_{2}(s)\right)^{2 n+1} d s, \\
& B(t)=B(0)-\int_{0}^{t} g(s) z_{1}(s)\left(A(s) z_{1}(s)+B(s) z_{2}(s)\right)^{2 n+1} d s .
\end{aligned}
$$

Using the fact that

$$
\begin{aligned}
\mid A(t) z_{1}(t)+B(t) z_{2}(t) & \left.\right|^{2 n+1} \\
& \leqq(|A(t)|+|B(t)|)^{2 n+1} \max \left[\left|z_{1}\right|^{2 n+1},\left|z_{2}\right|^{2 n+1}\right]
\end{aligned}
$$

it follows that 


$$
\begin{aligned}
|A(t)|+|B(t)| \leqq & |A(0)|+|B(0)| \\
& +2 \int_{0}^{t}|g(s)|(|A(s)|+|B(s)|)^{2 n+1} y(s) d s .
\end{aligned}
$$

Using Theorem 2, it follows that

$$
|A(t)|+|B(t)| \leqq \phi(t)
$$

where for $n \geqq 1$,

$$
\phi(t)=\left\{[|A(0)|+|B(0)|]^{-2 n}-4 n \int_{0}^{t}|g(s)| y(s) d s\right\}^{-1 / 2 n} .
$$

(The case $n=0$, treated by Trench, will not be developed here.)

Since the integral within the braces converges, an appropriate choice of initial conditions can guarantee that the expression in braces remains bounded away from zero by some fixed amount. Thus $\phi(t)$ is bounded and so then must the quantity $|A(t)|+|B(t)|$ be bounded. Using the boundedness of $A(t)$ and $B(t)$ it can be shown that the integrals on the right side of (5) are in fact convergent and thus $A(t)$ and $B(t)$ approach limits as $t \rightarrow \infty$.

Theorem 4. If $a(t)$ is continuous and if

$$
\int^{\infty}|a(t)| t^{2 n+2} d t<\infty
$$

then equation (1) has solutions which can be written

$$
x(t)=a t+b+o(1), \quad a, b \text { constants. }
$$

Proof. Set $f(t) \equiv 0$ in Theorem 3 and let $z_{1}(t)=t, z_{2}(t)=1$ be the solutions of $z^{\prime \prime}=0$. By Theorem 3 some solutions of equation (1) may be written as $x(t)=A(t) t+B(t)$ where $\lim _{t \rightarrow \infty} A(t)=a$ and $\lim _{t \rightarrow \infty} B(t)$ $=b, a, b$ constants. By observing that

$$
t|A(t)-a| \leqq \int_{t}^{\infty}|a(s)| s^{2 n+2}(|A(s)|+|B(s)|)^{2 n+2} d s
$$

it follows that

$$
t|A(t)-a|=o(1)
$$

and the proof is complete.

A related result for the linear case is given in [6, p. 239]. 


\section{REFERENCES}

1. R. A. Moore and Z. Nehari, Nonoscillation theorems for a class of nonlinear differential equations, Trans. Amer. Math. Soc. 93 (1959), 30-52.

2. F. V. Atkinson, On second order nonlinear oscillations, Pacific J. Math. 5 (1955), 643-647.

3. W. F. Trench, On the asymptotic behavior of solutions of second order linear differential equations, Proc. Amer. Math. Soc. 14 (1963), 12-14.

4. I. T. Kiguradze, On the asymptotic behavior of solutions of the equation $u^{\prime \prime}+a(t) u^{n}=0$, Math. Reviews 27 (1964), no. 386.

5. B. Viswanatham, A generalization of Bellman's lemma, Proc. Amer. Math. Soc. 14 (1963), 15-18.

6. E. Hille, Nonoscillation theorems, Trans. Amer. Math. Soc. 64 (1948), 234-252.

Sandia Laboratory, Albuquerque, New Mexico

\section{THE DOMAIN OF UNIVALENCE OF CERTAIN CLASSES OF MEROMORPHIC FUNCTIONS ${ }^{1}$}

\section{RAYMOND J. DISTLER}

1. Introduction. Let $K$ be a closed set of points in the complex plane and let $\mathcal{F}(K)$ be the family of functions

$$
f(z)=\sum_{k=1}^{n} \frac{A_{k}}{z-a_{k}}, \quad A_{k}>0, \quad k=1,2, \cdots, n,
$$

where all of the poles lie in $K$. In this paper we shall find the domain of univalence for the class $\mathcal{F}(K)$. By this we mean a domain $U=U(K)$ such that each $f(z) \in \mathcal{F}(K)$ is univalent in $U$, but if any open set is adjoined to $U$, then there is an $f_{0}(z) \in \mathcal{F}(K)$ that is not univalent in the enlarged domain.

In this direction Cakalov [2], [3] has proved two beautiful theorems.

THEOREM A. If all of the poles of (1) lie in the circle $|z| \leqq 1$, then $f(z)$ is univalent in the domain $|z|>\sqrt{ }(2)$, and this domain is maximal for the class of all such functions.

CoRollary. Let $\alpha(t), t \in[-\pi, \pi]$, be nondecreasing, with at least one point where it is increasing. Then

$$
f(z)=\int_{-\pi}^{\pi} \frac{d \alpha(t)}{z-e^{i t}}
$$

Presented to the Society, January 24, 1964; received by the editors July 5, 1963.

1 This paper is part of the author's dissertation done at the University of Kentucky. 